\title{
Innate Preferences Affect Results of Object Recognition Task in Wild Type and Alzheimer's Disease Mouse Models
}

\author{
Maria Rosaria Tropea ${ }^{\mathrm{a}}$, Giulia Sanfilippo ${ }^{\mathrm{a}}$, Federico Giannino ${ }^{\mathrm{a}}$, Valentina Davì ${ }^{\mathrm{a}}$, \\ Walter Gulisano ${ }^{\mathrm{a}}$ and Daniela Puzzo ${ }^{\mathrm{a}, \mathrm{b}, *}$ \\ ${ }^{a}$ Department of Biomedical and Biotechnological Sciences, Section of Physiology, University of Catania, \\ Catania, Italy \\ ${ }^{\mathrm{b}}$ Oasi Research Institute-IRCCS, Troina, Italy
}

Accepted 13 November 2021

Pre-press 17 December 2021

\begin{abstract}
.
Background: Object recognition task (ORT) is a widely used behavioral paradigm to assess memory in rodent models, due to its easy technical execution, the lack of aversive stressful stimuli, and the possibility to repeat the test on the same animals. However, mouse exploration might be strongly influenced by a variety of variables.

Objective: To study whether innate preferences influenced exploration in male and female wild type mice and the Alzheimer's disease (AD) model 3xTg.

Methods: We first evaluated how object characteristics (material, size, and shape) influence exploration levels, latency, and exploration modality. Based on these findings, we evaluated whether these innate preferences biased the results of ORT performed in wild type mice and $\mathrm{AD}$ models.

Results: Assessment of Exploration levels, i.e., the time spent in exploring a certain object in respect to the total exploration time, revealed an innate preference for objects made in shiny materials, such as metal and glass. A preference for bigger objects characterized by higher affordance was also evident, especially in male mice. When performing ORT, exploration was highly influenced by these innate preferences. Indeed, both wild type and AD mice spent more time in exploring the metal object, regardless of its novelty. Furthermore, the use of objects with higher affordance such as the cube was a confounding factor leading to "false" results that distorted ORT interpretation.

Conclusion: When designing exploration-based behavioral experiments aimed at assessing memory in healthy and AD mice, object characteristics should be carefully evaluated to improve scientific outcomes and minimize possible biases.
\end{abstract}

Keywords: Alzheimer's mice, exploration, object preference, object recognition task

\section{INTRODUCTION}

Memory is the faculty of our brain to store and recall information learned by experience during life,

\footnotetext{
*Correspondence to: Daniela Puzzo, Department of Biomedical and Biotechnological Sciences, Via Santa Sofia 87 - Torri Biologiche, 95123 Catania, Italy. Tel.: +39 095 4781322/35; E-mail: danypuzzo@yahoo.it.
}

and it is tightly related to learning, which is the ability to acquire new skills, actions, bearing, significance, and concepts. Different types of memory have been described and, among these, recognition memory is considered a subtype of declarative memory used to recognize already encountered items, people, and situations [1].

During the last decades, the study of recognition memory has gained the interest of several neuroscientists and a wide number of studies has been 
performed. A great contribution has been provided by studies in animal models such as rodents and non-human primates. However, while recognition memory can be accessible through oral and written language in humans, when using animal models an accurate ethological knowledge of the species is needed. In this context, behavioral paradigms aimed at investigating recognition memory are based on the spontaneous exploration of novel items. If two stimuli are simultaneously presented to a rat or a mouse and one of these two items was already experienced by the animal whereas the other one is new, the animal would spend more time exploring the novel item [2,3]. The Object Recognition Task (ORT) relies on this concept, and it is one of the most used behavioral paradigms to study recognition memory, its impairment in neurodegenerative disorders such as Alzheimer's disease (AD), and the efficacy of cognitive-enhancing drugs [4]. Different ORT paradigms have been adopted, but beside the specific protocol used, the test usually consists of two phases: 1) familiarization or training phase, in which the animal encounters two (or more) identical objects; 2) testing phase, in which the animal is exposed to one (or more) novel objects. The time spent exploring the familiar versus the novel object during the test phase is used as an index of recognition memory. Although ORT is relatively easy to perform, does not require expensive equipment nor a long animal training, or positive/negative reinforcement, several variables can influence the test risking to invalidate the results $[5,6]$. For example, recognition performance might be affected by the context [7], the exposure time to the objects during the familiarization phase or the delay between familiarization and test phase [3], the number and characteristics of presented objects [8-10] or their position [11], animal strain [12], and sex [13, 14]. Among these aspects, we focused on the characteristics of the objects used to evaluate memory in animal models of $\mathrm{AD}$. In fact, it frequently occurs that the difference in exploration of the familiar versus the novel object does not depend upon a difference in memory performance but on an innate preference for a certain object due to its intrinsic characteristics. If so, the exploration time will change a priori because of the higher attractive properties of the object and ORT results will be doubtful or not reliable. Here, we first studied whether exploration was influenced by exposure to objects of different material, size, and shape in wild type mice. Based on these findings, we evaluated how these innate preferences influenced ORT performances in male and female wild type mice and the $3 \times \mathrm{Tg}$ mouse model of AD.

\section{MATERIALS AND METHODS}

\section{Animals}

We used young male and female wild type mice (C57BL/6J) and $3 \times \mathrm{Tg}$ (APPSwe, PS1M146V, and tauP301L) AD mice [15] aged 7-8 months. Mice were kept in the animal facility of the University of Catania, maintained on a 12-h light/dark cycle (with lights on at $6.00 \mathrm{AM})$ in a temperature $\left(23 \pm 1^{\circ} \mathrm{C}\right)$ and humidity $(57 \pm 3 \%)$ controlled ventilated cabinet. Mice were housed in IVC standard cages enriched with a plastic object used as a nest and crease paper. Food (standard diet cubes) and water (filtered drinking water by particle filter, active carbon filter and UV light) were available ad libitum. The experiments complied with the ARRIVE guidelines, were carried out in accordance with the EU Directive 2010/63/EU for animal experiments, and received approval by the Institutional Animal Care and Use Committee.

\section{Object preference assessment}

Object preference assessment was performed by using an apparatus of our design and construction, consisting in an arena $(45 \times 45 \times 40 \mathrm{~cm})$ made by white matte polymethyl methacrylate non reflective panels. The floor of the arena was coated with a self-adhesive anti reflective film to further avoid reflections. Illumination was provided by a perpendicular diffused light source located $65 \mathrm{~cm}$ from the floor of the arena (Fig. 1). Based on preliminary studies, we chose to use two $6 \mathrm{~W}$ light bulbs providing 80 lux on the floor of the arena. Mice underwent a two-day period of habituation to the arena, where they were allowed to explore the environment for 5 min per day. Then, each mouse underwent four days of testing in a random order. The animal was put into the arena with three objects different in material, size, shape, and allowed to explore them for a total time of $10 \mathrm{~min}$. After each trial, the arena and the objects were cleaned with $70 \%$ ethanol and dried with absorbent paper. For each object we measured: 1) horizontal exploration, considered as the mouse pointing its nose toward the object at a distance not $>2 \mathrm{~cm}$, with the mouse standing with all four paws on the floor of the arena [t explor horiz (s)]; 2) vertical exploration, considered as the mouse exploring 



Fig. 1. Arena used to perform behavioral studies. A) Computer rendering of the apparatus. The arena is represented in a sectioned view to allow the visualization of objects placement. Light bulbs are inserted in a lid mounted perpendicular to the arena to ensure a diffuse and homogeneous illumination, avoiding object shadows as much as possible. B) Screenshot obtained from the recording camera placed on top of the arena.

the object with the two anterior paws lean on the object and the two posterior paws on the floor of the arena [t explor vert (s)]; 3) on-top exploration, considered as the mouse exploring the object standing on it [t explor on-top (s)]; 4) on-top non exploration, time spent sitting or standing on the object, without exploring it [t on top (s)]; 5) latency to first approach to each object [latency (s)]. Based on these measurements we calculated Exploration levels and Exploration type.

$$
\text { Exploration levels }=\frac{\mathrm{t} \text { explor }[\text { object } 1] \times 100}{\mathrm{t} \text { total }}
$$

where $\mathrm{t}=$ time and:

- $\mathrm{t}$ explor [object 1$]=\mathrm{t}$ explor horiz [object 1$](\mathrm{s})+$ $\mathrm{t}$ explor vert [object1] (s)+t explor on-top [object1] (s)

- $\mathrm{t}$ total $=\mathrm{t}$ explor [object 1$](\mathrm{s})+\mathrm{t}$ explor [object2] (s) $+\mathrm{t}$ explor [object3] (s)

- Exploration type: horizontal, vertical or on-top, where:

Horizontal Exploration type $=\frac{\mathrm{t} \text { explor horiz }[\text { object } 1] \times 100}{\mathrm{t} \text { explor }[\text { object } 1]}$

Vertical Exploration type $=\frac{\mathrm{t} \text { explor vert }[\text { object } 1] \times 100}{\mathrm{t} \text { explor }[\text { object } 1]}$

On top Exploration type $=\frac{\text { t explor ontop }[\text { object } 1] \times 100}{\mathrm{t} \text { explor [object } 1]}$
Mice with a total exploration time $<5 \mathrm{~s}$ were excluded [16].

\section{Choice of objects}

All the 3D printed objects were realized as previously described in polylactic acid (PLA) [17]. Briefly, after their design by a computer aided design software (Solidworks, France), 3D models were sliced and converted in g-code by a slicer software (Simplify3D, USA). For 3D printing we used a Prusa-inspired 3D printer customized according to our needs to obtain a $100 \mu \mathrm{m}$ resolution on z-axis and a printing nozzle diameter of $200 \mu \mathrm{m}$.

To assess the preference for materials, we used three cylindrical objects with similar size made by three different materials: glass, metal, and PLA. To assess the preference for size, we used three 3D printed cylinders made of PLA, with increasing size. The small object $(25 \times 22.5 \varnothing \mathrm{mm})$ measured half of the medium object $(50 \times 45 \emptyset \mathrm{mm})$, whereas the big object was one third bigger than the medium one $(75 \times 67.5 \varnothing \mathrm{mm})$. To assess the preference for shape, we used a pyramid, a cube, and a truncated sphere in PLA. The objects were designed to have comparable dimensions (mean height $=54 \pm 2.9 \mathrm{~mm}$ ).

\section{Object recognition task}

ORT test was performed as described previously [18] by using the same environmental conditions described for the Object Preference Assessment. 
After 3 days of habituation ( $5 \mathrm{~min} /$ day), mice underwent the training phase (T1). The mouse was put into the arena and allowed to explore two identical objects placed in the central part of the box, equally distant from the perimeter and the center for $10 \mathrm{~min}$. Twenty-four hours after T1, mice underwent the second trial (T2) to test memory retention for $10 \mathrm{~min}$. In $\mathrm{T} 2$, mice were presented with two different objects, respectively a "familiar" (i.e., the one used for T1) and a "novel" object. To understand whether innate preferences might influence memory, we alternated the use of preferred objects identified in the Object Preference Assessment. In different series of experiments, we used: 1) Familiar objects: two identical metal cylinders in T1; Novel object: PLA cylinder in T2; 2) Familiar objects: two identical PLA cylinders in T1; Novel object: metal cylinder in T2; 3) Familiar objects: two identical PLA cubes in T1; Novel object: PLA sphere in T2; 4) Familiar objects: two identical PLA spheres in T1; Novel object: PLA cube in T2; 5) Familiar objects: two identical PLA spheres in T1; Novel object: PLA pyramid in T2. Animal exploration was defined as the mouse pointing its nose toward the object from a distance not $<2 \mathrm{~cm}$. We calculated:

\section{Discrimination index $=$}

t explor [novel object](s) - t explor [familiar object](s) $\mathrm{t}$ explor [novel object](s)+t explor[familiar object](s)

Exploration was scored using a personal computer and mice with a total exploration time $<5 \mathrm{~s}$ were excluded [16].

\section{Statistical analysis}

Data were expressed as mean \pm standard error mean (SEM). Statistical analyses were performed by using Systat software (Chicago, IL, USA). We used: two-tailed $t$-test for comparisons between two groups, one-way ANOVA with Bonferroni post-hoc for comparisons among different groups, two-way ANOVA for interactions between variables. To assess if mice were able to discriminate between the two objects, we compared the performance obtained in our experimental groups with a fictive group (mean value of 10 independent experiments $=0$; standard error $=0.7$ ), as previously described [6].

The level of significance was set at $p<0.05$.

\section{RESULTS}

Assessment of preference in exploring objects of different materials

To investigate whether mice showed a preference for a certain material, they were exposed to three cylinders of similar size but different materials, i.e., metal, glass, and PLA (Fig. 2A), randomly positioned in the arena.

Exploration levels (time of exploration of each object/total exploration time) analyses showed that mice spent more time exploring glass $(43.21 \pm 2.04 \%$ total exploration time) and metal $(41.52 \pm 2.12 \%)$ objects in respect to PLA ones $(15.25 \pm 1.31 \%)$, as confirmed by ANOVA $\left(\mathrm{F}_{(2,57)}=70.74, p<0.0001\right.$; Bonferroni's $p=0$ for PLA versus glass or metal; Fig. 2B).

We then evaluated different types of explorations to understand the possible influence of object affordance on Exploration Levels. To this end, we distinguished among horizontal exploration, vertical exploration, and on-top exploration. We found that animals spent more time in exploring the three objects by horizontal exploration (one-way ANOVA $\mathrm{F}_{(2,57)}=63.609, p<0.0001$; Bonferroni's $p=0$ for horizontal exploration versus vertical or on-top), regardless the object material (two-way ANOVA exploration type*material interaction: $\mathrm{F}_{(4,171)}=0.055, p=0.994$; Fig. $2 \mathrm{C}$ ). To evaluate the affordance of the chosen objects, we scored the time spent on-top without exploring them (on-top non exploration time). We found that mice climbed on top of all the three objects, suggesting that the cylinder is an object with higher affordance. In particular, mice spent a higher percentage of non-exploration time on-top of the metal cylinder, reflecting a general preference for the metal material (metal: $42.01 \pm 6.44 \%$; glass: $29.91 \pm 5.36 \%$; PLA: $13.07 \pm 3.8 \% ; \mathrm{F}_{(2,57)}=7.47, p=0.001$; Bonferroni's $p=0.001$ for on-top non exploration between metal and PLA; Fig. 2D).

Finally, to evaluate if animals were particularly attracted by an object, we scored latency, i.e., how many seconds were needed for the mouse to approach a certain object for the first time. As expected, we found a lower latency for glass $(8.55 \pm 1.9 \mathrm{~s})$ and metal $(20.65 \pm 8.42 \mathrm{~s})$ in respect to PLA $(56.7 \pm 11.98 \mathrm{~s})$, suggesting that mice were preferentially attracted by these materials $\left(F_{(2,57)}=0.001\right.$; Bonferroni's $p=0.001$ and 0.012 for metal and glass versus PLA, respectively; Fig. 2E). 
A



B

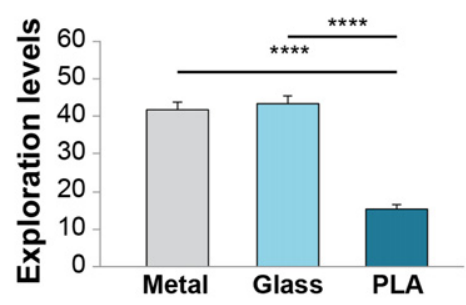

C

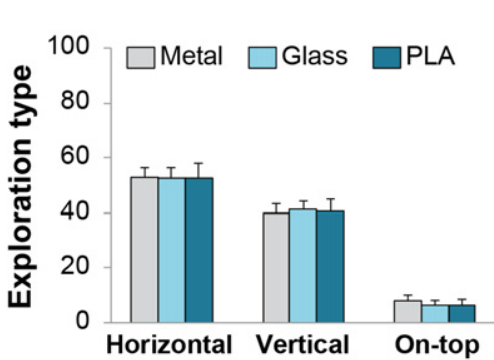

D

E
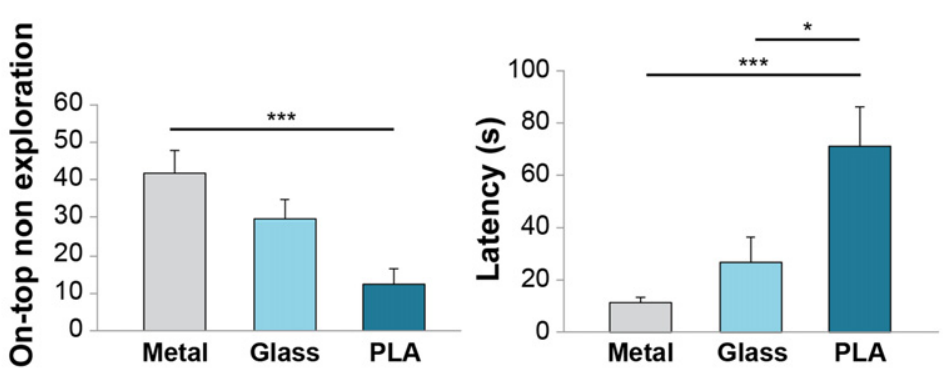

F
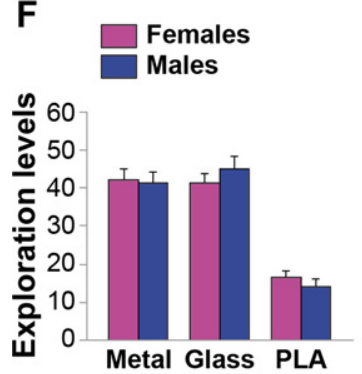

G

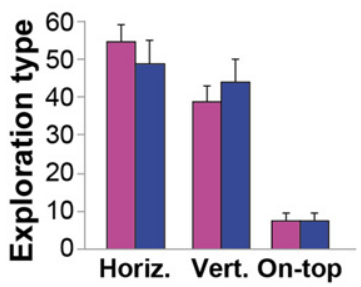

H

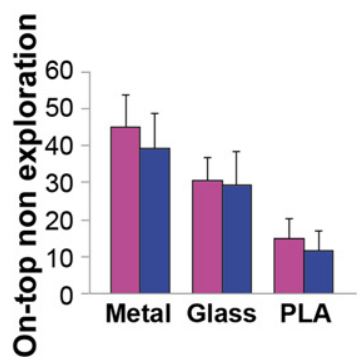

I

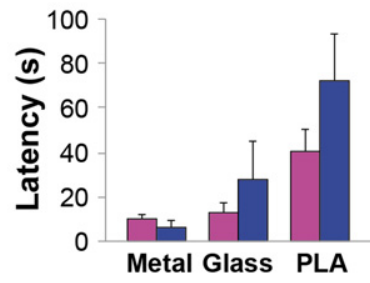

Fig. 2. Mice preferred to explore objects made in metal or glass. A) Metal, glass, and PLA objects used to evaluate preference for materials. B) Exploration levels (time of exploration of each object/total exploration time) was higher for metal and glass compared to PLA objects ( $n=20$ sex-balanced animals). C) Horizontal and vertical exploration were mainly used to approach the objects. Exploration modality was not affected by object material. D) Mice spent more time on top of the metal object. E) Latency to the first approach indicated that mice were preferentially attracted by metal and glass objects. F) Exploration levels as well as Exploration type G), On-top non exploration $\mathrm{H}$ ) and Latency for different materials I) did not change between males and females. Data expressed as mean \pm SEM. ${ }^{*} p<0.05,{ }^{* * *} p \leq 0.001$, $* * * * p \leq 0.0001$.

We then analyzed results in respect to sex, to evaluate possible gender differences. We found no differences between females and males in: 1) preferences for material (Two-way ANOVA sex*object material interaction: $\mathrm{F}_{(2,54)}=0.559, p=0.575$; Fig. 2F); 2) exploration type (Two-way ANOVA sex*exploration type interaction: $\mathrm{F}_{(2,54)}=0.868, p=0.425$; Fig. $\left.2 \mathrm{G}\right)$, 3 ) on-top non exploration (Two-way ANOVA sex*ontop non exploration interaction: $\mathrm{F}_{(2,54)}=0.384$, $p=0.683$; Fig. $2 \mathrm{H}$ ); 4) latency for different materials (Two-way ANOVA sex*latency interaction: $\mathrm{F}_{(2,54)}=1.161, p=0.321$; Fig. 2I).

\section{Assessment of preference in exploring objects of different size}

To investigate whether exploration might be influenced by object size, mice were exposed to three cylinders defined as small, medium, and big (Fig. 3A). Exploration levels analysis showed that mice preferentially explored the big object ( $46.37 \pm$ $1.4 \%$ total exploration time), followed by the medium size object $(37.7 \pm 1.58 \%)$, whereas they had little interest in the small one $(15.93 \pm 1.43 \%)$. Thus, preference paralleled the object size as confirmed 
A

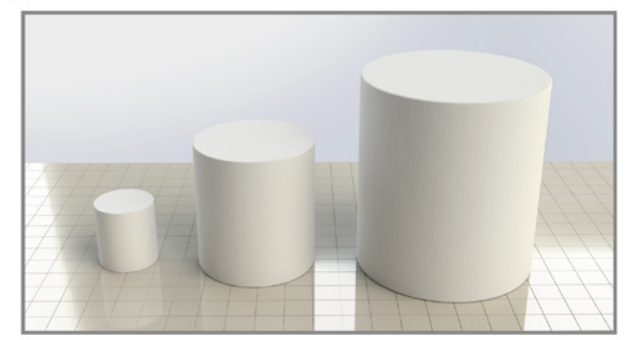

B



C

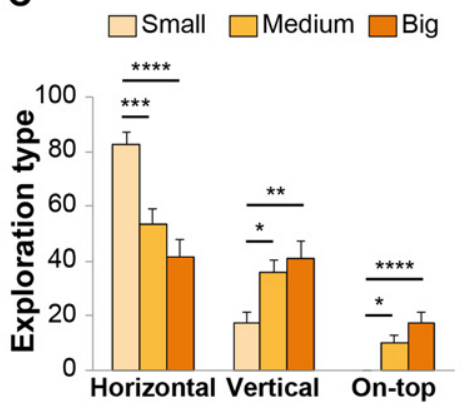

D

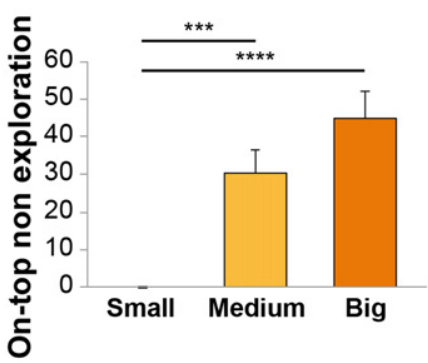

E

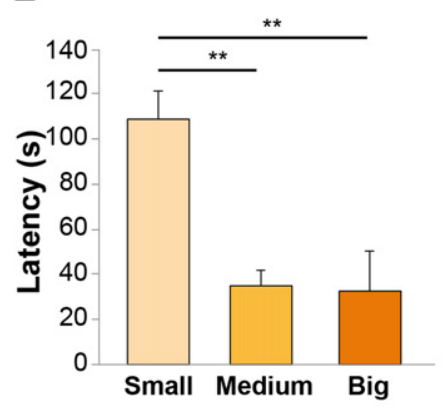

$\mathbf{F}$

Females

Males



G

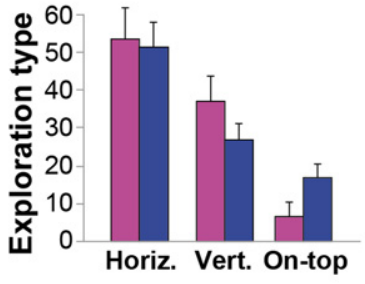

H

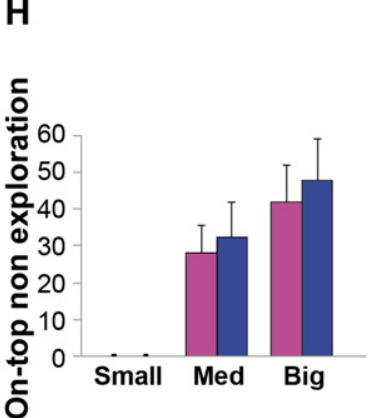

I

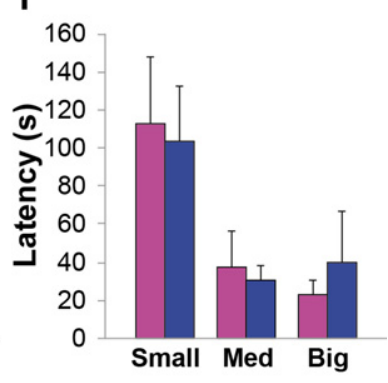

Fig. 3. Mice preferred to explore big and medium size objects. A) Small, medium, and big PLA cylinders to evaluate preference for size. B) Exploration levels indicated that mice spent more time exploring the big object followed by the medium size one $(n=20$ sex-balanced animals). C) Exploration type depended upon object size. The small object was mainly approached by horizontal exploration, the medium and especially big object also by vertical and on-top exploration. D) The medium and big objects were used for on-top non exploration. E) Latency to the first approach indicated that mice were preferentially attracted by medium and big objects. F) Total exploration time did not differ between males and females. F) Females equally explored the medium and big object, whereas males preferred the big object. G) No sex differences were recorded in exploration type, on-top non exploration $\mathrm{H}$ ), and Latency for different size objects I). Data expressed as mean \pm SEM. ${ }^{*} p<0.05,{ }^{* *} p \leq 0.01,{ }^{* * *} p \leq 0.001,{ }^{* * * *} p \leq 0.0001$.

by statistical analyses $\left(\mathrm{F}_{(2,57)}=112.762, p<0.0001\right.$; Bonferroni's $p<0.0001$ for big and medium versus small and big versus medium; Fig. 3B).

Object size also influenced exploration type (twoway ANOVA exploration type*material interaction: $\mathrm{F}_{(4,171)}=17.404, p<0.0001$; Fig. 3C). We found a significant difference between the modality used to explore the small object versus the medium and the big one (Fig. 3C). Horizontal modality was mainly dedicated to the small object $\left(\mathrm{F}_{(2,57)}=16.820\right.$, $p<0.0001$; Bonferroni's $p=0.001$ between small and medium; $p<0.0001$ between small and big; Fig. 3C), whereas vertical and on-top exploration to the medium and big object (vertical: $\mathrm{F}_{(2,57)}=6.594$, $p=0.003$; Bonferroni's $p=0.026$ between small and medium; $p=0.003$ between small and big; ontop: $\mathrm{F}_{(2,57)}=9.37, p<0.0001$; Bonferroni's $p=0.038$ between small and medium; $p<0.0001$ between small and big; Fig. 3C). Also, the big object was equally explored by horizontal and vertical modality 
(Fig. 3C). Analyses of on-top non exploration confirmed this difference, due to the fact that mice could only climb bigger objects, being unable to sit on the small one (small: no time spent on-top; medium: $30.2 \pm 5.93 \%$; big: $44.25 \pm 7.37 \% ; \mathrm{F}_{(2,57)}=17.489$, $p<0.0001$; Bonferroni's $p=0.001$ between small and medium; $p<0.0001$ between small and big; Fig. 3D).

The preference for objects with higher dimensions and affordance was confirmed by the lower latency for the medium and big object (small: 108.35 \pm 12.9 s; medium: $34.2 \pm 6.65 \mathrm{~s}$; big: $31.7 \pm 18.86 \mathrm{~s} ; \mathrm{F}_{(2,57)}$ $=7.18, p=0.002$; Bonferroni's $p=0.006$ and $p=$ 0.005 for small versus medium and big, respectively; Fig. 3E).

Analyses of performance in female and male mice confirmed that object size influenced exploration time, although a sex-related difference was found (Two-way sex*object size interaction: $\mathrm{F}_{(2,54)}=8.056$, $p=0.001$; Fig. $3 \mathrm{~F}$ ). In particular, both females and males preferred to explore the medium and the big object compared to the small one. However, females spent more time than males in exploring the medium object ( $p=0.004$; Fig. 3F), whereas males spent more time than females in exploring the big object ( $p=0.036$; Fig. $3 \mathrm{~F}$ ). Conversely, no sex differences were found when analyzing exploration type, as both females and males preferred horizontal exploration (Two-way sex*exploration type interaction: $\mathrm{F}_{(2,54)}=1.324, p=0.274$; Fig. 3G). Concerning on-top non exploration, both sexes preferred the medium and big object not being able to climb the small one (Two-way ANOVA sex*on-top non exploration interaction: $\mathrm{F}_{(2,54)}=0.072, p=0.931$; Fig. $\left.3 \mathrm{H}\right)$. Latency was not different in males and females (Twoway ANOVA sex ${ }^{*}$ latency interaction: $\mathrm{F}_{(2,54)}=0.191$, $p=0.827$; Fig. 3I).

\section{Assessment of preference in exploring objects of different shapes}

To assess whether shape might influence preference and exploration modality, mice were exposed to three objects of similar size, material (PLA) and color (grey), but different shape, i.e., a pyramid, a cube, and a truncated sphere (Fig. 4A).

Exploration levels analysis showed that mice preferentially explored the pyramid $(37.20 \pm 2.22 \%$ total exploration time) and the cube $(41.45 \pm 2.76 \%$ total exploration time) in respect to the truncated sphere $(21.33 \pm 2.02 \%$ total exploration time $)$ $\left(\mathrm{F}_{(2,57)}=20.2, p<0.0001\right.$; Bonferroni's $p<0.0001$ comparing sphere with pyramid or cube; Fig. 4B).
Animals preferred the horizontal exploration modality compared to the vertical or the on-top exploration $\left(\mathrm{F}_{(2,57)}=39.299, \quad p<0.0001\right.$; Bonferroni's $p<0.0001$ comparing horizontal versus vertical or on-top; Fig. 4C). The preference for horizontal exploration was evident for all of the three objects, whereas on-top exploration was mainly dedicated to the cube, due to the difficulty in climbing the pyramid or the truncated sphere $\left(\mathrm{F}_{(2.57)}=23.749, p<0.0001\right.$; Bonferroni's $p<0.0001$ comparing cube versus pyramid or sphere; Fig. 4C). This was confirmed by analyses of on-top non exploration (pyramid: no time spent on-top; cube: $47 \pm 8.07 \%$; truncated sphere: $4.08 \pm 1.87 \% ; \mathrm{F}_{(2,57)}=29.608, p<0.0001$; Bonferroni's $p<0.0001$ comparing cube versus pyramid or sphere; Fig. 4D). Finally, objects' shape did not influence latency (pyramid: $25.85 \pm 10.42 \%$; cube: $24.75 \pm 6.02 \%$; truncated sphere: $13.09 \pm 3.47 \%$; $\mathrm{F}_{(2,57)}=0.833, p=0.44$; Fig. 4E).

No differences were found between males and females in preference for different shapes (Twoway ANOVA sex*object shape interaction: $\mathrm{F}_{(2,54)}=$ $3.031, p=0.057$; Fig. 4F). Analyses of results showed a difference in exploration type between females and males (Two-way ANOVA sex*exploration type interaction: $F_{(2,54)}=6.732, p=0.002$; Fig. $\left.4 G\right)$. In particular, females preferred horizontal exploration $(p<0.0001$; Fig. $4 \mathrm{G})$, whereas males spent a similar amount of time in exploring objects by horizontal and vertical exploration ( $p=1$; Fig. $4 \mathrm{G}$ ). However, male mice showed a higher on-top non exploration for the cube compared with females $(p=0.038$; Fig. 4G). No differences were found in latency (Twoway ANOVA sex*latency interaction: $\mathrm{F}_{(2,54)}=0.064$, $p=0.871$; Fig. 4I).

\section{Innate object preference prejudiced ORT results in wild type and Alzheimer's disease mice}

Based on the Object Preference assessment, we investigated how the choice of objects influenced results of exploration-based behavioral test commonly used to investigate memory. To this end, we performed ORT in wild type and AD mice to evaluate recognition memory by assessing the differences in exploration of a familiar versus a novel object [4, 18]. In particular, we exposed mice to the objects toward which mice showed an innate preference and to neutral objects. We selected the most representative objects of a certain category and showed an example of ORT performed by using two objects with an 
A

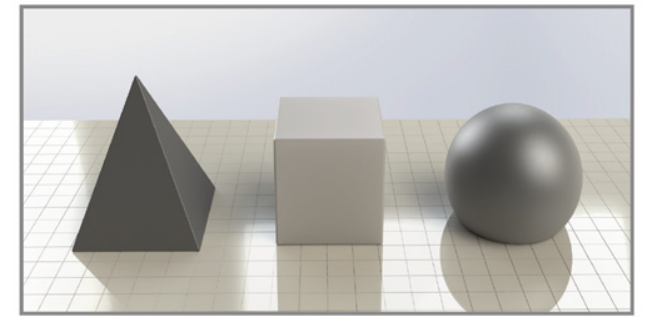

B

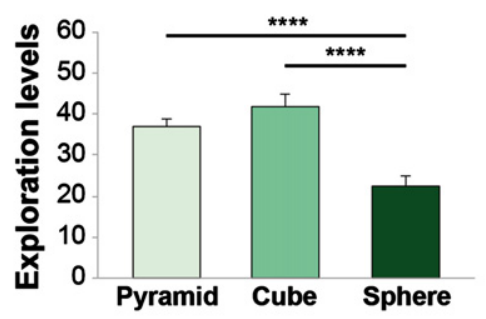

E

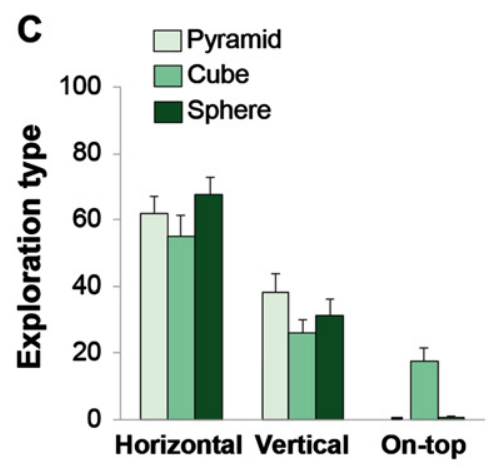

D


F

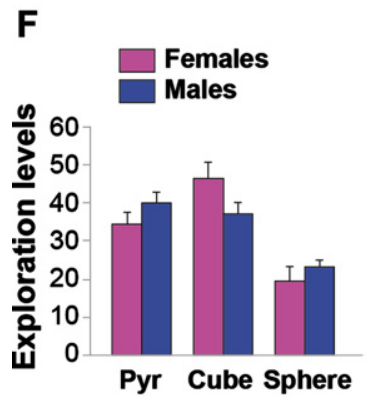

G

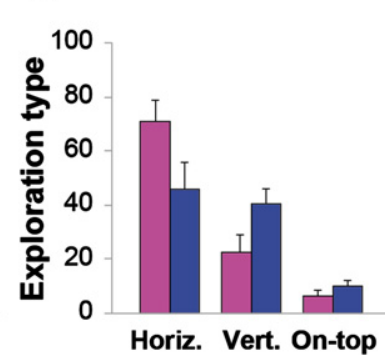

H

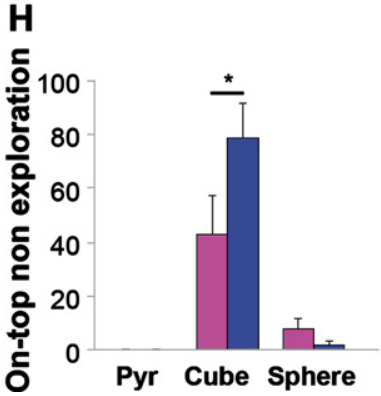

I

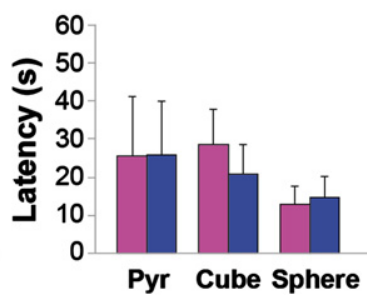

Fig. 4. Mice preferred to explore objects with higher affordance. A) PLA pyramid, cube, and truncated sphere to evaluate preference for different shapes. B) Exploration levels indicated that mice spent more time exploring objects with higher affordance such as the pyramid and the cube ( $n=20$ sex-balanced animals). C) Horizontal and vertical exploration were mainly used to approach the objects and were not influenced by object shape. On-top exploration and D) On-top non exploration was essentially performed for the cube. E) Latency to the first approach was similar for the three objects. E) Exploration levels and Exploration type G) did not significantly differ between males and females. However, females preferred horizontal versus vertical exploration, whereas males equally explored object by horizontal and vertical exploration. H) On-top non exploration for the cube was higher in males. I) Latency for different shapes did not change between males and females. Data expressed as mean \pm SEM. ${ }^{*} p<0.05,{ }^{* * * *} p \leq 0.0001$.

evident difference in brightness (metal versus PLA) or affordance (cube versus sphere).

In a series of experiments, we evaluated the impact of objects made by different materials. During the training phase (T1), mice were exposed to two identical metal cylinders for $10 \mathrm{~min}$. After a 24-h interval, mice were exposed to a metal cylinder (familiar object) and PLA cylinder (novel object) (Fig. 5A). We found that both wild type and AD mice spent a similar amount of time in exploring the familiar and the novel object during the testing phase ( $t$-test wild type: $\mathrm{t}_{(18)}=1.274, p=0.219 ;$ AD: $\mathrm{t}_{(18)}=0.818$, $p=0.424$ between familiar and novel object; Fig. 5B). Analyses of Discrimination Index confirmed that there was no difference between wild type and $\mathrm{AD}$ mice $\left(0.09 \pm 0.07\right.$ versus $0.06 \pm 0.07 ; \mathrm{t}_{(18)}=0.282$; $p=0.781$; Fig. 5C) and that both genotypes were (apparently) unable to learn since Discrimination Index was not different than zero when compared with the fictive group ( $t$-test wild type: $\mathrm{t}_{(18)}=0.936$, $p=0.362 ;$ AD: $\mathrm{t}_{(18)}=0.673, p=0.510 ;$ Fig. 5C). No differences were recorded in total exploration time $\left(\mathrm{t}_{(18)}=0.445, p=0.662 ;\right.$ Fig. 5D). Males and females of both experimental groups behaved similarly when 
analyzing the time spent in exploring the familiar and novel object (Two-way ANOVA sex*familiarnovel object interaction in wild type: $\mathrm{F}_{(1,16)}=1.020$, $p=0.328$; in AD mice: $\mathrm{F}_{(1,16)}=0.052, p=0.822$; Fig. 5E) and Discrimination Index (sex*genotype interaction: $\mathrm{F}_{(1,16)}=0.104, p=0.751$; Fig. 5F).

Thus, in this experimental condition, wild type did not present the increase in exploration of the novel object expected when memory is intact.

Conversely, when we used two identical PLA cylinders as familiar objects during the training phase, and a PLA cylinder paired with a metal cylinder as a novel object during the testing phase (Fig. 4G), both wild type and $\mathrm{AD}$ mice spent more time in exploring the novel object ( $t$-test wild type: $\mathrm{t}_{(18)}=6.256$, $p<0.0001 ;$ AD: $\mathrm{t}_{(18)}=3.363, p=0.003$ between familiar and novel object; Fig. 5H). These results were confirmed by analyses of Discrimination Index that suggested no significant differences between wild type and $\mathrm{AD}$ performances $(0.36 \pm 0.08$ versus $0.20 \pm 0.08 ; \mathrm{t}_{(18)}=1.320 ; p=0.203$; Fig. 5I). Notably, both genotypes resulted able to learn as Discrimination Index was different than zero when compared with the fictive group ( $t$-test wild type: $\mathrm{t}_{(18)}=3.372, p=0.003 ;$ AD: $\mathrm{t}_{(18)}=2.363, p=0.030$; Fig. 5I). Total exploration time did not differ between the two genotypes $\left(\mathrm{t}_{(18)}=0.750 ; p=0.463\right.$; Fig. 5J). No gender differences were found in time spent exploring the familiar versus novel object (Twoway ANOVA sex*familiar-novel object interaction in wild type: $\mathrm{F}_{(1,16)}=0.004, p=0.952$; in AD mice: $\mathrm{F}_{(1,16)}=0.008, p=0.929$; Fig. $\left.5 \mathrm{~K}\right)$ or Discrimination Index (sex* genotype interaction: $\mathrm{F}_{(1,16)}=0.486$, $p=0.496$; Fig. 5L).

Thus, in this experimental condition, AD mice did not present the expected impairment of memory. Overall, these experiments suggested that the use of an attractive metal object either used as familiar or novel object prejudiced results of ORT that erroneously indicated an impairment of memory in wild type mice or a normal memory in AD mice.

In a second series of experiments, we evaluated whether objects of different shapes might influence ORT. During the training phase mice were exposed to two identical PLA cubes (Fig. 6Aa). As expected, no differences were found in exploration levels of the two cubes (wild type: $\mathrm{t}_{(22)}=1.465, p=0.154$; AD: $\mathrm{t}_{(22)}=1.794, p=0.087$ between familiar and novel object; Fig. 6B) or in total exploration time $\left(\mathrm{t}_{(22)}=0.294, p=0.771\right.$; Fig. 6C). After $24 \mathrm{~h}$, mice were exposed to a PLA sphere as a novel object (Fig. 6Ab). We found that both genotypes spent a higher amount of time in exploring the familiar object ( $t$-test wild type: $\mathrm{t}_{(22)}=9.435, p<0.0001 ;$ AD: $\mathrm{t}_{(18)}=2.937, p=0.008$ between familiar and novel object; Fig. 6D), with no differences in total exploration time $\left(\mathrm{t}_{(22)}=0.392 ; p=0.699\right.$; Fig. 6E). Discrimination Index confirmed that wild type and $\mathrm{AD}$ mice behaved similarly, both preferring the exploration of the familiar object $(-0.32 \pm 0.04$ versus $-0.17 \pm 0.08 ; \mathrm{t}_{(22)}=1.613, p=0.121$; Fig. $\left.6 \mathrm{~F}\right)$. This was particularly evident for wild type mice that showed a Discrimination Index different than zero but negative (comparison with the fictive group: $t$-test wild type: $\mathrm{t}_{(20)}=2.913, p=0.001$; Fig. $6 \mathrm{~F}$ ). No differences were found in males and females in time spent exploring the familiar versus the novel object (Twoway ANOVA sex*familiar-novel object interaction in wild type: $\mathrm{F}_{(1,20)}=0.602, p=0.447$; in AD mice: $\mathrm{F}_{(1,20)}=0.023, p=0.882$; Fig. $\left.6 \mathrm{G}\right)$, as confirmed by the analyses of Discrimination Index (sex* genotype interaction: $\mathrm{F}_{(1,20)}=0.136, p=0.716$; Fig. $6 \mathrm{H}$ ).

This result was opposite to that normally expected, since wild type mice should dedicate more time in exploring the novel object, whereas $\mathrm{AD}$ mice, due to their memory deficit, usually spent the same amount of time in exploring the familiar and the novel object. Thus, in this experimental setting, the use of objects with higher affordance such as the cube during the training phase did not allow a correct evaluation of memory.

We then inverted the objects and used two identical PLA spheres as familiar objects and a PLA cube as a novel object (Fig. 6Ib-c). Also in this case, no differences were found in Exploration levels of the two spheres during the training phase (wild type: $\mathrm{t}_{(22)}=1.432, p=0.166 ;$ AD: $\mathrm{t}_{(22)}=1.921, p=0.368$ between familiar and novel object; Fig. $6 \mathrm{~J}$ ) or in total exploration time $\left(\mathrm{t}_{(20)}=0.021, p=0.983\right.$; Fig. $\left.6 \mathrm{~K}\right)$. However, a higher total exploration time in $\mathrm{T} 1$ was recorded in wild type mice exposed to cubes (Fig. 6C) in respect to spheres (Fig. 6K) $\left(\mathrm{t}_{(22)}=3.037\right.$, $p=0.006$ ).

During the training phase, wild type mice spent a higher $\%$ of time in exploring the novel object $\left(t\right.$-test: $\left.\mathrm{t}_{(22)}=5.322, p<0.0001\right)$, whereas AD mice dedicated the same amount of time in exploring the two objects $\left(\mathrm{t}_{(20)}=0.814, p=0.425\right.$ between familiar and novel object; Fig. 6L). Total exploration time was unchanged $\left(\mathrm{t}_{(21)}=0.081 ; p=0.936\right.$; Fig. $\left.6 \mathrm{M}\right)$. Analyses of Discrimination Index confirmed the difference between wild type and AD mice $(0.27 \pm 0.07$ versus $\left.0.04 \pm 0.08 ; \mathrm{t}_{(21)}=2.136, p=0.045\right)$ and that only wild type mice were able to learn ( $t$-test 

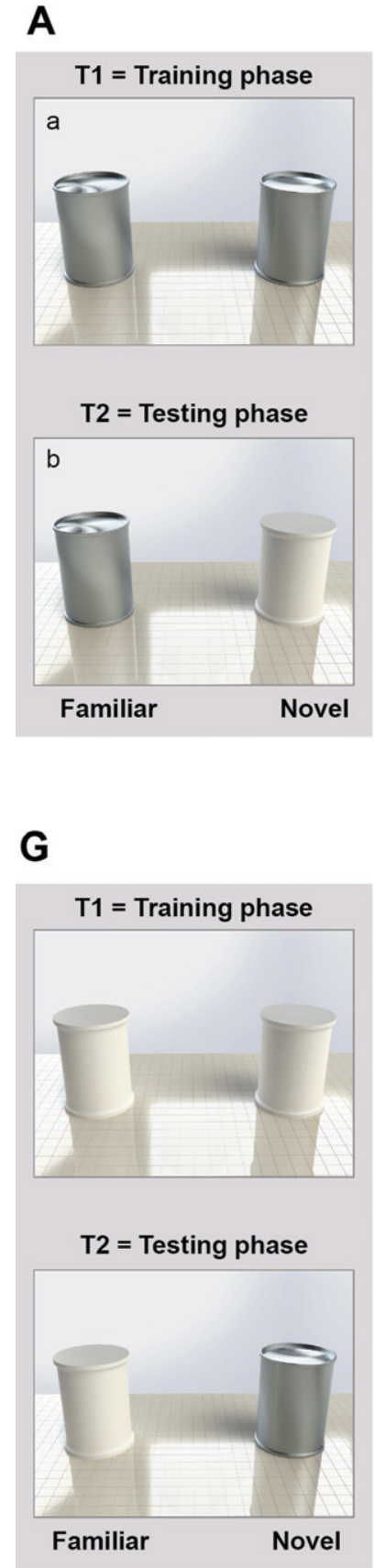

\section{B}

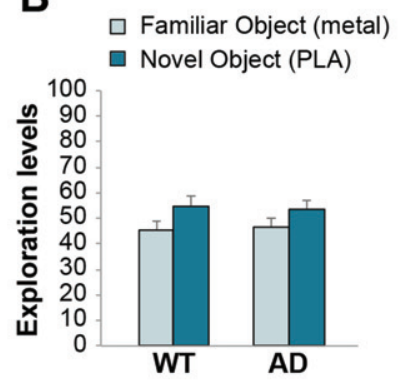

\section{E}

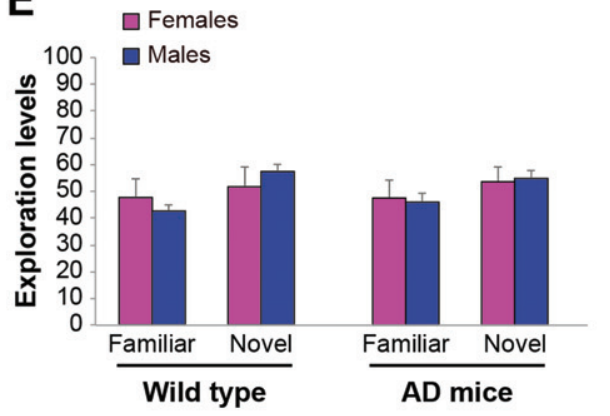

C

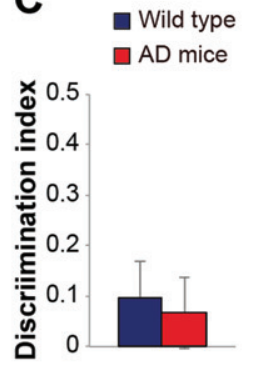

$\mathbf{F}$
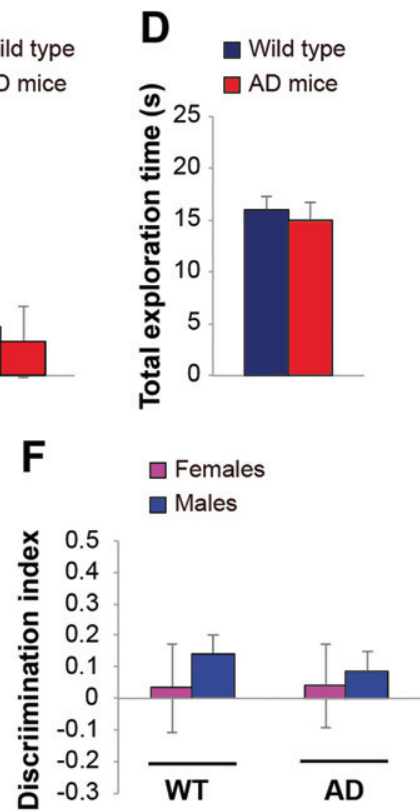

H


K

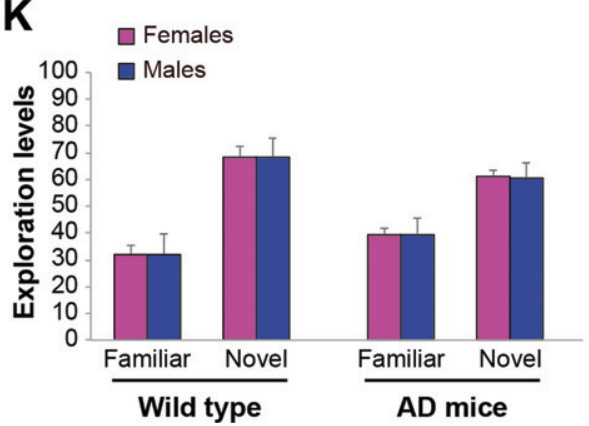

I

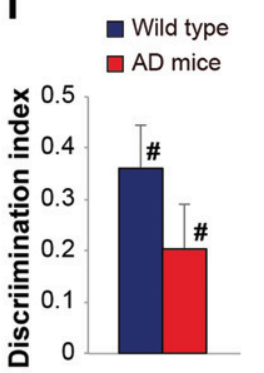

$\mathbf{J}$


Fig. 5. The use of metal objects biased results of object recognition task in wild type and AD mice. A) Objects used during the T1 training phase (a) and the T2 testing phase (b). B) Both wild type and AD mice dedicated the same amount of time in exploring the familiar and the novel object ( $n=10 / 10$ sex-balanced animals). C) Discrimination Index indicated an impairment of recognition memory in both wild type and AD mice. D) Total exploration time was similar in wild type and AD mice. E) Males and females of both genotypes spent a similar amount of time in exploring the familiar and the novel object; F) No gender differences were found in Discrimination Index of wild type and $\mathrm{AD}$ mice. $\mathrm{G}$ ) Objects used during the $\mathrm{T} 1$ training phase (a) and the $\mathrm{T} 2$ testing phase (b) in another series of experiments. $\mathrm{H}$ ) Both wild type and $\mathrm{AD}$ mice dedicated a higher amount of time in exploring the novel object ( $n=10 / 10$ sex-balanced animals). I) Discrimination Index indicated a normal recognition memory in both wild type and AD mice. J) Total exploration time was similar in wild type and AD mice. K) No gender differences were found in exploration of novel versus familiar object and L) Discrimination Index in wild type and AD mice. Data expressed as mean \pm SEM. ${ }^{* * *} p<0.005,{ }^{* * * *} p \leq 0.0001$, \# difference with zero (comparison with fictive group). 
A



B



C



D

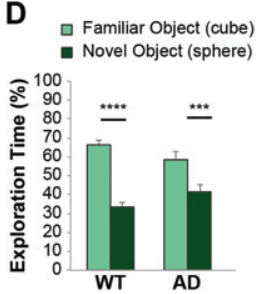

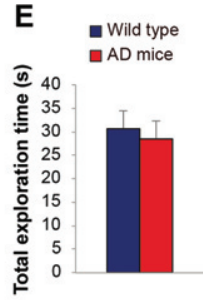
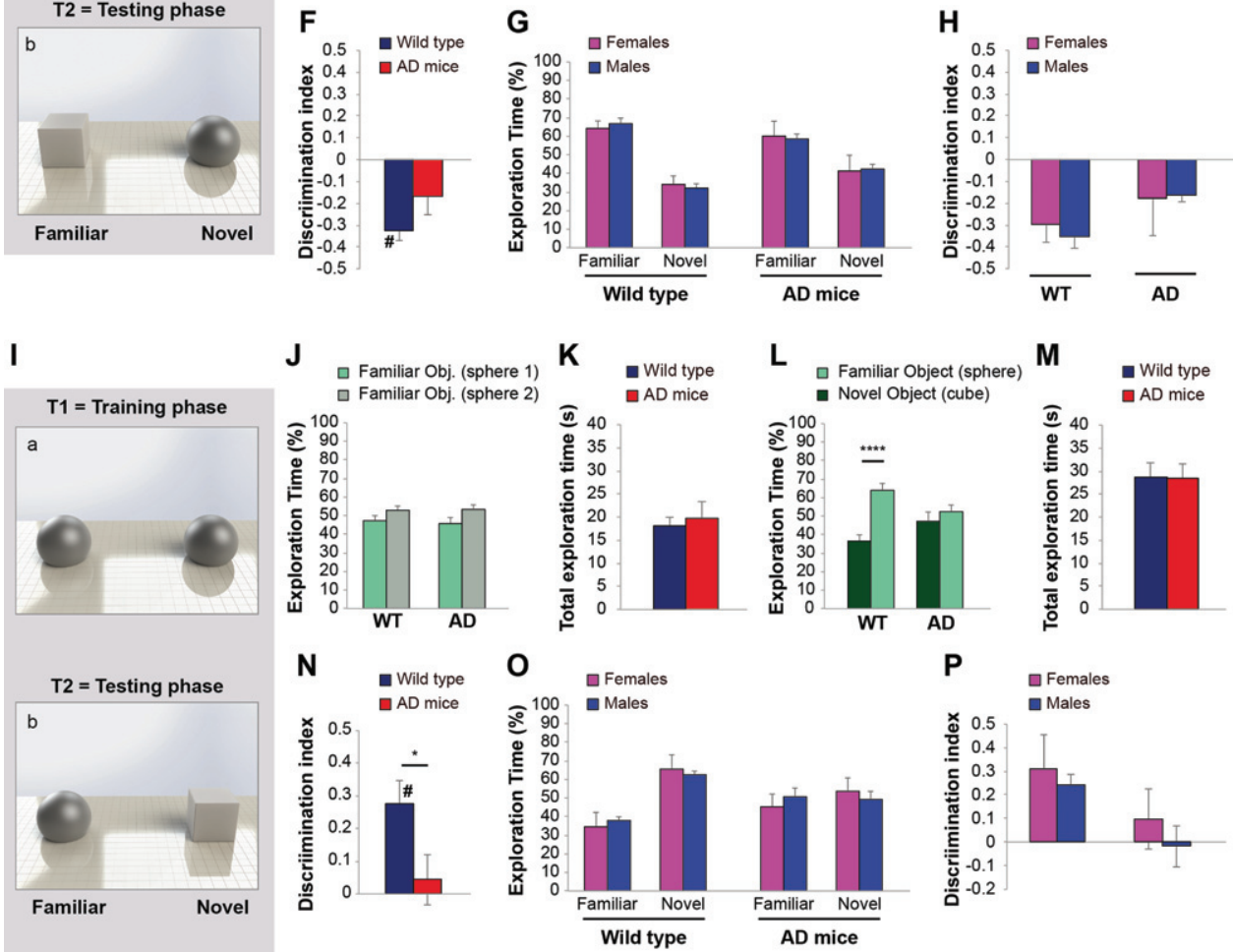

잉

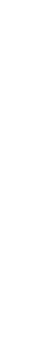


versus fictive group wild type: $\mathrm{t}_{(20)}=2.695, p=0.014$; AD: $\mathrm{t}_{(19)}=0.429, p=0.673$; Fig. $6 \mathrm{~N}$ ). No gender differences were found in exploration (sex*familiarnovel object interaction in wild type: $\mathrm{F}_{(1,20)}=0.356$, $p=0.558$; in AD mice: $\mathrm{F}_{(1,18)}=1.026, p=0.325$; Fig. 60) and Discrimination Index (sex*genotype interaction: $\mathrm{F}_{(1,19)}=0.056, p=0.816$; Fig. $\left.6 \mathrm{P}\right)$.

Thus, when we used the sphere in the training phase and the cube in the testing phase, ORT results allowed to distinguish between a normal cognition in wild type animals and the expected impairment in AD mice.

Even if this choice of objects could be appropriate, considering that the use of objects with higher affordance such as the cube might represent a bias, we performed another series of experiments maintaining the sphere as a familiar object and substituting the cube with the pyramid as a novel object (Fig. 6Ic). These experiments confirmed that the use of neutral objects is the most appropriate as wild type mice spent more time in exploring the novel object, i.e. the pyramid, compared to the familiar object ( $t$-test: $\mathrm{t}_{(18)}=10.318, p<0.0001$; Fig. 6Q), whereas AD mice dedicated a similar amount of time in exploring the two objects ( $t$-test: $\mathrm{t}_{(18)}=0.691, p=0.498$; Fig. 6Q). The Discrimination Index was different between wild type and $\mathrm{AD}$ mice $(0.30 \pm 0.03$ versus $-0.03 \pm 0.07$; $t$-test: $\mathrm{t}_{(18)}=3.914, p=0.001$; Fig. $\left.6 \mathrm{R}\right)$, and indicated that only wild type animals were able to learn ( $t$-test versus fictive group wild type: $\mathrm{t}_{(18)}=3.775$, $p=0.001 ;$ AD: $\mathrm{t}_{(18)}=0.363, p=0.721$; Fig. $\left.6 \mathrm{R}\right)$. No differences were recorded in total exploration time $\left(\mathrm{t}_{(18)}=0.487 ; p=0.632\right.$; Fig. $\left.6 \mathrm{~S}\right)$ nor gender differences in exploration levels (sex* familiar-novel object interaction in wild type: $\mathrm{F}_{(1,16)}=0.246, p=0.627$; in AD mice: $\mathrm{F}_{(1,16)}=2.428, p=0.139$; data not shown) and Discrimination Index (sex*genotype interaction: $\mathrm{F}_{(1,16)}=0.605, p=0.448$; Fig. $\left.6 \mathrm{~T}\right)$.

Thus, the use of neutral objects allowed distinguishing between normal and impaired memory in wild type and $\mathrm{AD}$ mice, avoiding the possible biases due to the use of attractive objects toward which mice manifest an innate preference.

\section{DISCUSSION}

Exploration is an innate behavior aimed at providing information about the environment and particular items or individuals. Given its ubiquitous nature across species, exploratory behavior has been considered a powerful tool to study cognitive and affective performance in pre-clinical research. A variety of tests aimed to investigate memory are based on novelty exploration [19]. Several objects, such as pyramids, parallelepipeds, cubes, hemispheres, truncated pyramids but even complex objects such as coffee mugs, cans, Playmobil and Lego toys, PVC pipes, glass vases and candlesticks, have been used to perform ORT or other exploration-based studies in rodents [20]. The choice of materials is also variable, since objects used can be made of glass, porcelain, metal, plastic, rubber, and wood [20].

In this manuscript, we have confirmed that intrinsic object qualities might influence exploration behavior and affect the results of test aimed at investigating memory in animal models.

We found that shining objects made of metal or glass elicited a greater curiosity in mice, consistent with previous studies $[10,20]$. In fact, although rats and mice have limited color vision and show poor color discrimination, they are able to discriminate between stimuli that differ in brightness [21, 22]. This implies that, when using these objects to perform ORT or similar studies, the experimenter might not distinguish between memory performance and the innate attraction towards shiny objects. Our results demonstrated that when pairing a metal with a PLA object, ORT results were clearly biased. When the metal cylinder was used either as a familiar or a novel object, both wild type and AD mice spent more time in exploring it during the testing phase. Therefore, if a shiny object is used as a familiar object, one can wrongly interpret that wild type mice had memory impairment. Conversely, if it is used as a novel object, results might be read as AD mice having a normal memory. Thus, the use of metal objects is not recommended, especially if paired with plastic ones. It would be more appropriate to randomly alternate metal and glass as familiar or novel objects, and not to use them together with neutral objects made in plastic or, as in our experiments, in PLA. Otherwise, PLA objects might be used alone, considering that total exploration time is maintained in an acceptable range and that, if brilliant objects are not present during the test, mice will spend more time in exploring PLA objects, as previously demonstrated [18].

When studying exploration behavior related to objects of different size, we found that the objects with big and medium dimensions appeared more suitable for exploration, even if the bigger object was mainly used for climbing or sitting. Conversely, the small object resulted the less explored and attractive. This is in line with previous studies suggesting 
that object size influenced exploration and preference [23] probably because an object with a similar size to the animal, results in a higher affordance. Indeed, affordance is referred to what animals could perceive, recognize, and memorize of an object [10], and depends upon the relationship between the properties of objects and the abilities of animals [24]. Therefore, affordance is influenced by those characteristics of the object that attract the animal because of a possible interaction with it [25]. In general, an object with higher affordance is the one that the mouse could lean on, climb onto, grasp, or perform other actions belonging to common rodent activities, or an object able to elicit sensory perceiving. Consistently, our findings showed that the small object, approachable only by horizontal exploration, was less explored in respect to the medium and the big object that could be also explored by vertical and on-top exploration. Consequently, if the aim of the study is to evaluate memory, it would be better to choose an object with high affordance that mice are interested to explore but with characteristics that do not allow an excessive active interaction to avoid distraction and a decrease of exploration time. In agreement with previous findings, a medium size object, i.e., comparable to mouse dimension, should be preferentially chosen [26], and, in any case, it would be better not to associate objects of different size in the same experimental protocol.

Concerning possible sex-differences, we found that females equally explored the medium and the big object, whereas males preferred the big object. Although we cannot explain this different behavior, we can speculate that it might depend upon the mouse size, being males bigger than females (mean weight $=33 \pm 2$ versus $26 \pm 2$ grams).

Affordance also addressed the preference related to the object shape. Previous studies have demonstrated that mice explored objects that can be climbed significantly longer than objects that can only be touched [10]. In our experimental setting, mice mainly explored the cube and the pyramid, compared to the truncated sphere. However, they spent a higher amount of time sitting on the cube that was used as a sort of "observation tower" to explore the surrounding environment.

This behavior was particularly evident in male mice and influenced exploration type. In fact, while female behavior mirrored the common exploration type preference (horizontal $>$ vertical $>$ ontop), males displayed no differences between horizontal and vertical exploration, and spent more time standing on the cube to visualize the surrounding environment. This might be related to sex differences in navigation and object exploration $[1,2]$. In fact, it has been previously demonstrated that females navigate using both landmarks and Euclidean geometry, preferentially encoding information based on objects characteristics and locations, whereas males are inclined to use geometric cues using the shape of the environment as a reference $[3,5,6]$.

Here, the use of objects with high affordance such as the cube affected ORT results especially in wild type mice that spent a very high amount of time exploring the cube during the testing phase. This resulted in a clear inversion of Discrimination Index that indicates memory impairment. Furthermore, the higher time spent in exploring the familiar cube versus the novel object, determined a false alteration of novelty preferences, which is a characteristic of autism spectrum disorders and other stereotyped behaviors [27]. This bias was avoided by using a different object that, although with good affordance, cannot be climbed, i.e., the pyramid.

In conclusion, in line with previous findings, we have shown that intrinsic characteristics of the object used affect total exploration time, latency, recognition and Discrimination Index. Objects in PLA, with a similar size to that of the animal and shapes preserving a good affordance resulted more appropriate to perform behavioral tests based on exploration. These findings should be considered when performing memory tests based on novelty to provide reliable data.

\section{ACKNOWLEDGMENTS}

This work was supported by University of Catania intramural funds (Progetto Piaceri) to D.P.

Authors' disclosures available online (https:// www.j-alz.com/manuscript-disclosures/21-5209r1).

\section{REFERENCES}

[1] Squire LR, Wixted JT, Clark RE (2007) Recognition memory and the medial temporal lobe: A new perspective. Nat Rev Neurosci 8, 872-883.

[2] Eichenbaum H, Yonelinas AP, Ranganath C (2007) The medial temporal lobe and recognition memory. Ann Rev Neurosci 30, 123-152.

[3] Ennaceur A, Delacour J (1988) A new one-trial test for neurobiological studies of memory in rats. 1: Behavioral data. Behav Brain Res 31, 47-59.

[4] Zhang R, Xue G, Wang S, Zhang L, Shi C, Xie $\times(2012)$ Novel object recognition as a facile behavior test for evaluating drug effects in A $\beta P P / P S 1$ Alzheimer's disease mouse model. J Alzheimers Dis 31, 801-812. 
[5] Ameen-Ali KE, Easton A, Eacott MJ (2015) Moving beyond standard procedures to assess spontaneous recognition memory. Neurosci Biobehav Rev 53, 37-51.

[6] Akkerman S, Prickaerts J, Steinbusch HWM, Blokland A (2012) Object recognition testing: Statistical considerations. Behav Brain Res 232, 317-322.

[7] Piterkin P, Cole E, Cossette M-P, Gaskin S, Mumby DG (2008) A limited role for the hippocampus in the modulation of novel-object preference by contextual cues. Learn Mem 15, 785-791.

[8] Oliveira AMM, Hawk JD, Abel T, Havekes R (2010) Post-training reversible inactivation of the hippocampus enhances novel object recognition memory. Learn Mem 17, $155-160$.

[9] Hale G, Good M (2005) Impaired visuospatial recognition memory but normal object novelty detection and relative familiarity judgments in adult mice expressing the APPswe Alzheimer's disease mutation. Behav Neurosci 119, 884-891.

[10] Ennaceur A (2010) One-trial object recognition in rats and mice: Methodological and theoretical issues. Behav Brain Res 215, 244-254.

[11] Williams MT, Herring NR, Schaefer TL, Skelton MR, Campbell NG, Lipton JW, McCrea AE, Vorhees CV (2007) Alterations in body temperature, corticosterone, and behavior following the administration of 5-methoxydiisopropyltryptamine ('Foxy') to adult rats: A new drug of abuse. Neuropsychopharmacology 32, 1404-1420.

[12] Şík A, van Nieuwehuyzen P, Prickaerts J, Blokland A (2003) Performance of different mouse strains in an object recognition task. Behav Brain Res 147, 49-54.

[13] Frick KM, Gresack JE (2003) Sex differences in the behavioral response to spatial and object novelty in adult C57BL/6 mice. Behav Neurosci 117, 1283-1291.

[14] Bettis T, Jacobs LF (2012) Sex differences in object recognition are modulated by object similarity. Behav Brain Res 233, 288-292.

[15] Oddo S, Caccamo A, Shepherd JD, Murphy MP, Golde TE, Kayed R, Metherate R, Mattson MP, Akbari Y, LaFerla FM (2003) Triple-transgenic model of Alzheimer's disease with plaques and tangles. Neuron 39, 409-421.
[16] Gulinello M, Mitchell HA, Chang Q, O'Brien WT, Zhou Z, Abel T, Wang L, Corbin JC, Veeraragavan S, Samaco RC, Andrews NA, Fagiolini M, Cole TB, Burbacher TM, Crawley JN (2019) Rigor and reproducibility in rodent behavioral research. Neurobiol Learn Mem 165, 106780.

[17] Gulisano W, Tropea MR, Arancio O, Palmeri A, Puzzo D (2018) Sub-efficacious doses of phosphodiesterase 4 and 5 inhibitors improve memory in a mouse model of Alzheimer's disease. Neuropharmacology 138, 151-159.

[18] Gulisano W, Melone M, Ripoli C, Tropea MR, Li Puma DD, Giunta S, Cocco S, Marcotulli D, Origlia N, Palmeri A, Arancio O, Conti F, Grassi C, Puzzo D (2019) Neuromodulatory action of picomolar extracellular A 342 oligomers on presynaptic and postsynaptic mechanisms underlying synaptic function and memory. J Neurosci 39, 5986-6000.

[19] Leger M, Quiedeville A, Bouet V, Haelewyn B, Boulouard M, Schumann-Bard P, Freret T (2013) Object recognition test in mice. Nature Prot 8, 2531-2537.

[20] Antunes M, Biala G (2012) The novel object recognition memory: Neurobiology, test procedure, and its modifications. Cogn Process 13, 93-110.

[21] Jacobs GH, Fenwick JA, Williams GA (2001) Cone-based vision of rats for ultraviolet and visible lights. $J$ Exp Biol 204, 2439-2446.

[22] Jacobs GH (1993) The distribution and nature of colour vision among the mammals. Biol Rev 68, 413-471.

[23] Buccafusco JJ (2000) Methods of behavior analysis in neuroscience. 1st ed. CRC Press/Taylor \& Francis, Boca Raton.

[24] Chemero A, Heyser C (2005) Object exploration and a problem with reductionism. Synthese 147, 403-423.

[25] Blaser R, Heyser C (2015) Spontaneous object recognition: A promising approach to the comparative study of memory. Front Behav Neurosci 9, 183.

[26] Levin ED, Buccafusco JJ (2006) Introduction. In Animal Models of Cognitive Impairment, Levin ED, Buccafusco JJ, eds. CRC Press/Taylor \& Francis, Boca Raton.

[27] Jacob S, Landeros-Weisenberger A, Leckman JF (2009) Autism spectrum and obsessive-compulsive disorders: OC behaviors, phenotypes and genetics. Autism Res 2, 293-311. 